BULL. AUSTRAL. MATH. SOC.

VOL. 18 (1978), 21-28.

\title{
A new graph product and its spectrum
}

\section{C.D. Godsil and B.D. McKay}

\begin{abstract}
A new graph product is introduced, and the characteristic polynomial of a graph so-formed is given as a function of the characteristic polynomials of the factor graphs. A class of trees produced using this product is shown to be characterized by spectral properties.
\end{abstract}

\section{Notation and preliminaries}

All graphs considered in this paper are finite, and without loops and multiple or directed edges. Any undefined graph-theoretical terms will have the meanings given to them in Behzad and Chartrand [1].

If $G$ is a graph with adjacency matrix $A(G)$, then we denote the characteristic polynomial $\operatorname{det}(\lambda I-A(G))$ of $A(G)$ by $G(\lambda)$, and refer to it as the characteristic polynomial of $G$. If $G$ is a rooted graph then we denote by $G^{\prime}$ the graph obtained from $G$ when the root vertex is removed. The characteristic polynomial of the rooted graph $G$ is just the characteristic polynomial of the unrooted graph with the same vertex and edge sets as $G$.

DEFINITION 1.1. Let $H$ be a labelled graph on $n$ vertices. Let $G$ be a sequence of $n$ rooted graphs $G_{1}, G_{2}, \ldots, G_{n}$. Then by $H(G)$ we denote the graph obtained by identifying the root of $G_{i}$ with the $i$ th vertex of $H$. We call $H(G)$ the rooted product of $H$ by $G$.

Figure 1 illustrates this construction with $H$ the path on three

Received 14 September 1977. 
vertices and $G$ consisting of three copies of the rooted path on two vertices

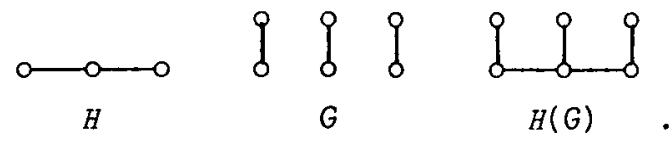

FIGURE 1

DEFINITION 1.2. Given a labelled graph $H$ on $n$ vertices and a sequence $G$ of $n$ rooted graphs, we define the matrix $A_{\lambda}(H, G)$ as follows :

$$
A_{\lambda}(H, G)=\left(a_{i j}\right)
$$

where

$$
a_{i j}= \begin{cases}G_{i}(\lambda), & i=j, \\ -h_{i j} G_{i}^{\prime}(\lambda), & i \neq j,\end{cases}
$$

and $A(H)=\left(h_{i j}\right)$ is the adjacency matrix of $H$.

If, for example, $B$ and $G$ are represented in Figure 1, then $A_{\lambda}(H, G)$ is the matrix

$$
\left[\begin{array}{ccc}
\lambda^{2}-1 & -\lambda & 0 \\
-\lambda & \lambda^{2}-1 & -\lambda \\
0 & -\lambda & \lambda^{2}-1
\end{array}\right] .
$$

\section{The polynomial of the rooted product}

In this section we prove the following:

THEOREM 2.1. $H(G)(\lambda)=\operatorname{det} A_{\lambda}(H, G)$.

This result has already been proved by Schwenk [4] in the case where $G$ consists of $n$ isomorphic rooted graphs. The method we use to prove the result in general is quite different from his, however.

We will need the following lemma. 
LEMMA 2.2. Let $K$ and $L$ be rooted graphs, and let $K \cdot L$ denote the graph obtained by identifying the roots of $K$ and $L$. Then

$$
K \cdot L(\lambda)=K(\lambda) L^{\prime}(\lambda)+K^{\prime}(\lambda) L(\lambda)-\lambda K^{\prime}(\lambda) L^{\prime}(\lambda) \text {. }
$$

Proof. See Schwenk [4], or Godsil and McKay [2].

Proof of Theorem 2.1. We will use induction on the number of vertices of $H(G)$. Suppose this number is $N$, and that the theorem holds for all labelled graphs $H$ and sequences $G$ such that $H(G)$ has less than $N$ vertices. For $n=1$, the theorem follows from the definition of $A_{\lambda}(H, G)$, so we assume $n \geq 2$.

Let $F$ denote the sequence of rooted graphs obtained from $G$ by replacing the graph $G_{n}$ by $K_{1}$, the graph with only one vertex. $F^{\prime}$ will be used to denote the subsequence of $G$ consisting of the graphs $G_{1}, G_{2}, \ldots, G_{n-1}$. Let $H^{\prime}$ denote the graph obtained from $H$ by deleting the vertex labelled $n$, and let $H(F)^{\prime}$ denote the graph obtained from $H(F)$ be deleting the vertex which was labelled $n$ in $H$. Clearly $H(F)^{\prime}=H^{\prime}\left(F^{\prime}\right)$. The situation is represented diagrammatically in Figure 2.
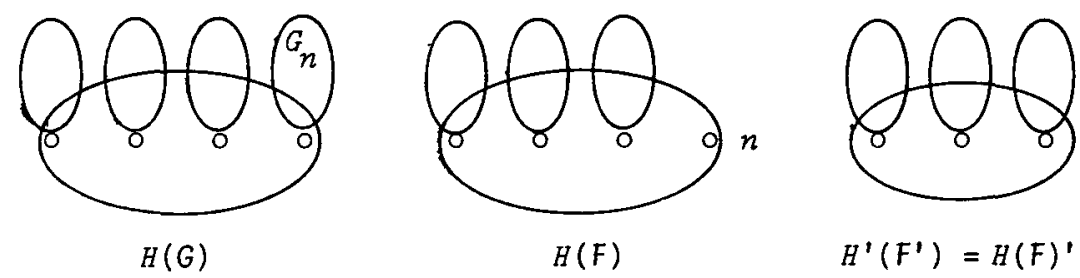

FIGURE 2

It follows at once from Lemma 2.2 that

$$
H(G)(\lambda)=G_{n}(\lambda) H(F)^{\prime}(\lambda)+G_{n}^{\prime}(\lambda) H(F)(\lambda)-\lambda G_{n}^{\prime}(\lambda) H(F)^{\prime}(\lambda) .
$$

Now 


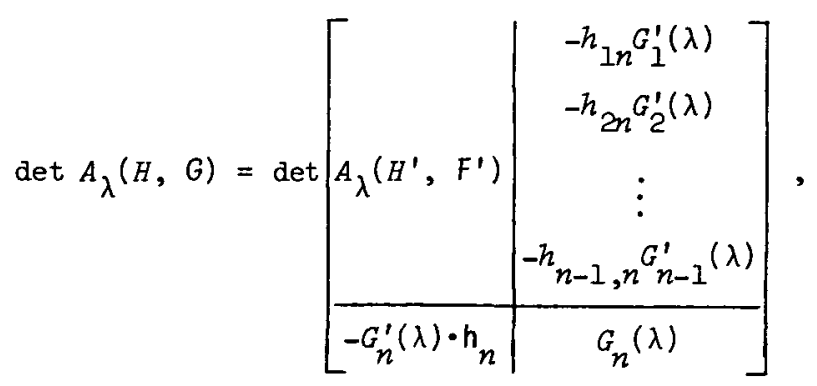

where $h_{n}$ denotes the row vector $\left(h_{n 1}, h_{n 2}, \ldots, h_{n, n-1}\right)$. Since the determinant of a matrix is a linear function of any row, the right side of (2) can be expressed as

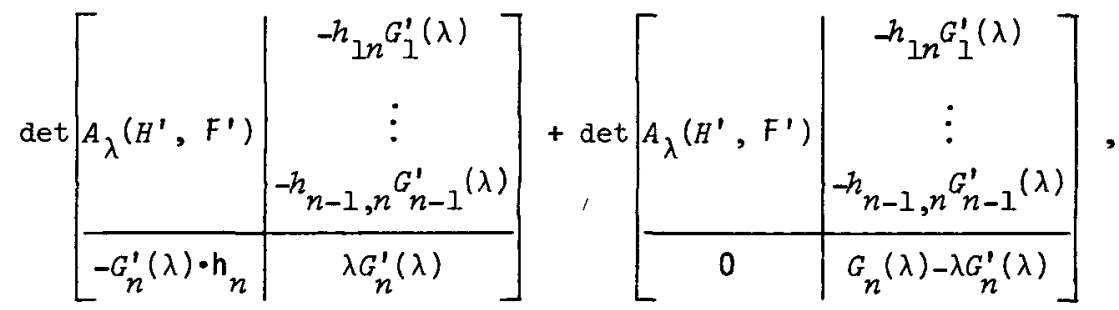

which equals

$$
G_{n}^{\prime}(\lambda) \operatorname{det} A_{\lambda}(H, F)+\left(G_{n}(\lambda)-\lambda G_{n}^{\prime}(\lambda)\right) \operatorname{det} A_{\lambda}\left(H^{\prime}, F^{\prime}\right) .
$$

By our induction hypothesis $\operatorname{det} A_{\lambda}\left(H^{\prime}, F^{\prime}\right)=H^{\prime}\left(F^{\prime}\right)(\lambda)$, and $\operatorname{det} A_{\lambda}(H, F)=H(F)(\lambda)$. Hence (3) may be rewritten as

$$
G_{n}^{\prime}(\lambda) H(F)(\lambda)+G_{n}(\lambda) H^{\prime}\left(F^{\prime}\right)(\lambda)-\lambda G_{n}^{\prime}(\lambda) H^{\prime}\left(F^{\prime}\right)(\lambda) .
$$

Since $H^{\prime}\left(F^{\prime}\right)=H(F)^{\prime}$, a comparison of (4) with (I) shows that we have established the theorem.

We note that on dividing the $i$ th row of $A_{\lambda}(H, G)$ by $G_{i}^{\prime}(\lambda)$ for $i=1,2, \ldots, n$, one obtains a matrix of the form $\Lambda-A(H)$, where

$$
\Lambda=\operatorname{diag}\left\{\frac{G_{1}(\lambda)}{G_{1}^{\prime}(\lambda)}, \frac{G_{2}(\lambda)}{G_{2}^{\prime}(\lambda)}, \ldots, \frac{G_{n}(\lambda)}{G_{n}^{\prime}(\lambda)}\right\} .
$$

Hence

$$
\operatorname{det} A_{\lambda}(H, G)=\operatorname{det}(\Lambda-A(H)) \cdot \prod_{i=1}^{n} G_{i}^{\prime}(\lambda) .
$$


In the special case where the $G_{i}$ are all isomorphic, $\Lambda=\left(G_{1}(\lambda) / G_{1}^{\prime}(\lambda)\right) I$ and so

$$
H(G)(\lambda)=G_{1}^{\prime}(\lambda)^{n} H\left(\frac{G_{1}(\lambda)}{G_{1}^{\prime}(\lambda)}\right\} .
$$

This is the formula given in [4].

Finally if $G$ consists of $n$ copies of $P_{2}$, the path on two vertices, one obtains, from (6),

$$
H(G)(\lambda)=\lambda^{n} H\left(\lambda-\frac{1}{\lambda}\right),
$$

since $P_{2}(\lambda)=\lambda^{2}-1$, and $P_{2}^{\prime}(\lambda)=\lambda$. We will use (7) in the next section.

\section{A spectral characterization of a class of trees}

NOTATION 3.1. A matching of a graph $T$ is a set of mutually nonadjacent edges. An m-matching consists of $m$ such edges, A matching $M$ such that every vertex of $T$ is an end vertex of some edge in $M$ is called a l-factor.

We recall, from [3] for example, that if $T$ is a tree on $n$ vertices, then

$$
T(\lambda)=\sum_{m=0}^{[n / 2]}(-1)^{m} a_{2 m} \lambda^{n-2 m},
$$

where $a_{2 m}$ is the number of m-matchings of $T$.

We will use $T\left(P_{2}\right)$ to denote the rooted product of $T$ by the collection consisting of one copy of $P_{2}$ for each vertex of $T$. It follows from (8) that, if $T$ is a tree on $n$ vertices, then $T(-\lambda)=(-1)^{n} T(\lambda)$, and so from $(7)$ above we find

$$
\begin{aligned}
T\left(P_{2}\right)\left(\frac{\bar{I}}{\lambda}\right) & =\left(\frac{-1}{\lambda}\right)^{n} T\left(\lambda-\frac{1}{\lambda}\right) \\
& =(-1)^{n} \lambda^{-2 n} T\left(P_{2}\right)(\lambda) .
\end{aligned}
$$


We will call a polynomial of degree $2 n$ satisfying (9) symmetric.

THEOREM 3.2. Let $T$ be a tree on $2 n$ vertices. Then $T(\lambda)$ is symmetric if and only if $T=S\left(P_{2}\right)$ for some tree $S$.

Proof. The sufficiency follows from the remarks above. We give the proof of the necessity in a number of steps.

We assume $n \geq 2$. Let $a_{2 m}$ denote the number of m-matchings of $T \cdot$

(a) $T$ has 1 -matching and $2 n-1$ (n-1)-matchings.

Since $T(\lambda)$ is symmetric we have $a_{0}=a_{2 n}$ and $a_{2}=a_{2 n-2}$. But $a_{0}=1$ and $a_{2}$ is just the number of edges of $T$ and so the claim follows.

(b) $T$ has $n$ end vertices.

Let $M$ be the $n$-matching of $T$. By counting $(n-1)$-matchings we will show that every edge in $M$ contains an end vertex of $T$.

Say that an $(n-1)$-matching $N$ is of type $I$ if it is a subset of $M$. Clearly there are $n$ such matchings.

Let $v_{2} v_{3}$ be an edge of $T$ not in $M$. Then there are vertices $v_{1}$ and $v_{4}$ of $T$ such that both $v_{1} v_{2}$ and $v_{3} v_{4}$ lie in $M$. Let $N$ be the (n-1)-matching obtained from $M$ by replacing $v_{1} v_{2}$ and $v_{3} v_{4}$ by the edge $v_{2} v_{3}$. We will call $N$ a type II (n-1)-matching. The number of type II (n-1)-matchings is just the number of edges of $T$ not in $M$. This equals $n-1$.

Since a type II $(n-1)$-matching is not a subset of $M$ we have already found $2 n-1$ distinct $(n-1)$-matchings.

Let $v_{3} v_{4}$ be an edge in $M$ such that neither $v_{3}$ nor $v_{4}$ is an end-vertex. Let $v_{2}$ and $v_{5}$ be vertices of $T$ adjacent to $v_{3}$ and $v_{4}$ respectively. Then there exist vertices $v_{1}$ and $v_{6}$ in $T$ such that $v_{1} v_{2}$ and $v_{5} v_{6}$ lie in $M$. Replacing the edges $v_{1} v_{2}, v_{3} v_{4}$, and $v_{5} v_{6}$ of $M$ by the edges $v_{2} v_{3}$ and $v_{4} v_{5}$ we obtain an $(n-1)$-matching $N$. 
Since $|N \cap M| \leq n-3, N$ is not of type I or II.

Thus the existence of an edge $v_{3} v_{4}$ in $M$ such that neither $v_{3}$ nor $v_{4}$ is an end vertex of $T$ implies that $T$ has at least $2 n(n-1)$ matchings. Hence every edge in $M$ contains at least one end vertex of $T$. If some edge in $M$ consisted of two adjacent end-vertices, then $T$ would be disconnected. Therefore $T$ must have exactly $|M|=n$ end vertices.

(c) $T=S\left(P_{2}\right)$ for some tree $S$.

Let $S$ be the tree obtained by removing the $n$ end vertices from $T$. As $T$ has a 1-factor, it cannot have a vertex adjacent to two end vertices. Hence $T=S\left(P_{2}\right)$.

We remark that the proof of the theorem actually shows that a tree on $2 n$ vertices with an $n$-matching, and $2 n-1(n-1)$-matchings is a rooted product.

Note that Theorem 3.2 does not hold when the assumption that $T$ is a tree is dropped. For example the graph shown in Figure 3 is obviously not a rooted product, although its characteristic polynomial is $\lambda^{8}-9 \lambda^{6}+16 \lambda^{4}-9 \lambda^{2}+1$, which is symmetric.

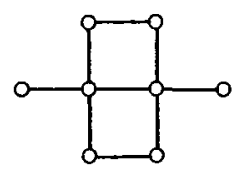

FIGURE 3

\section{References}

[1] Mehdi Behzad, Gary Chartrand, Introduction to the theory of graphs (Allyn and Bacon, Boston, 1971).

[2] C. Godsil and B. McKay, "Some computational results on the spectra of graphs", Combinatorial Mathematics IV, 73-92 (Proc. Fourth Austral. Conf., University of Adelaide, 1975. Lecture Notes in Mathematics, 560. Springer-Verlag, Berlin, Heidelberg, New York, 1976). 
[3] Horst Sachs, "Beziehungen zwischen den in einem Graphen enthal tenen Kreisen und seinem charakteristischen Polynom", Publ. Math. Debrecen 11 (1964), 119-134.

[4] Allen J. Schwenk, "Computing the characteristic polynomial of a graph", Graphs and combinatorics, 153-172 (Proc. Capital Conf. Graph Theory and Combinatorics, George Washington University, 1973. Lecture Notes in Mathematics, 406. Springer-Verlag, Berlin, Heidelberg, New York, 1974).

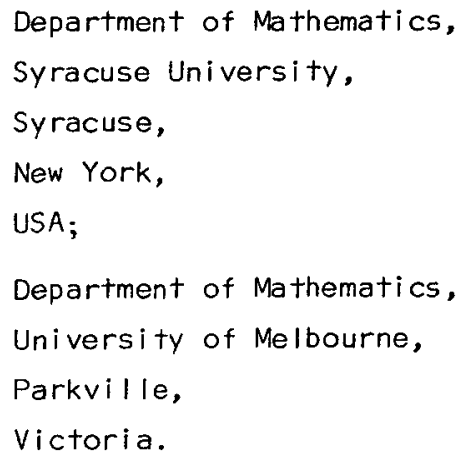

